ARTICLE

\title{
Stable high-capacity and high-rate silicon-based lithium battery anodes upon two-dimensional covalent encapsulation
}

\author{
Xinghao Zhang ${ }^{1,2}$, Denghui Wang ${ }^{1,2}$, Xiongying Qiu', Yingjie Ma1, Debin Kong ${ }^{1}$, Klaus Müllen³, \\ Xianglong Li(i) ${ }^{1,2}$ \& Linjie Zhi (i) ${ }^{1,2}$ 凶
}

Silicon is a promising anode material for lithium-ion and post lithium-ion batteries but suffers from a large volume change upon lithiation and delithiation. The resulting instabilities of bulk and interfacial structures severely hamper performance and obstruct practical use. Stability improvements have been achieved, although at the expense of rate capability. Herein, a protocol is developed which we describe as two-dimensional covalent encapsulation. Twodimensional, covalently bound silicon-carbon hybrids serve as proof-of-concept of a new material design. Their high reversibility, capacity and rate capability furnish a remarkable level of integrated performances when referred to weight, volume and area. Different from existing strategies, the two-dimensional covalent binding creates a robust and efficient contact between the silicon and electrically conductive media, enabling stable and fast electron, as well as ion, transport from and to silicon. As evidenced by interfacial morphology and chemical composition, this design profoundly changes the interface between silicon and the electrolyte, securing the as-created contact to persist upon cycling. Combined with a simple, facile and scalable manufacturing process, this study opens a new avenue to stabilize silicon without sacrificing other device parameters. The results hold great promise for both further rational improvement and mass production of advanced energy storage materials.

\footnotetext{
${ }^{1}$ CAS Key Laboratory of Nanosystem and Hierarchical Fabrication, CAS Center for Excellence in Nanoscience, National Center for Nanoscience and Technology, Beijing 100190, China. ${ }^{2}$ University of Chinese Academy of Sciences, Beijing 100049, China. ${ }^{3}$ Max Planck Institute for Polymer Research, Mainz

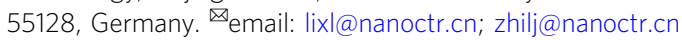


T $o$ meet the ever-demanding performance requirements of lithium-ion batteries (LIBs) and post-lithium rechargeable batteries for applications such as powering electric vehicles and integrating intermittent renewable energy, high-capacity electrochemically active electrode materials are being extensively exploited $^{1-8}$. The binding between such electrode materials and the adjacent electrically conductive media (e.g., carbon black) and consequently the electrode framework is a critical issue ${ }^{9-12}$, in particular when employing conventional electrode formulation with known conductive additives and binders ${ }^{13}$. This electrical connection, at the same time, must be resistant to the electrolyte of a battery cell. The high-capacity active materials inevitably suffer from large volume changes during charging and discharging processes (Fig. 1a). Thus, silicon possesses the highest theoretical gravimetric (specific) capacity, which is ten times that of commercial graphite ( $372 \mathrm{mAh} \mathrm{g}^{-1}$ ), but experiences up to $300 \%$ volume change upon lithiation and delithiation ${ }^{6,14}$. Such a large volume change causes pulverization and electrical disconnection of the active material ${ }^{15}$, but also forms dynamic interfaces ${ }^{9}, 16-19$. This "dynamic interfacing" is prone of worsening or blocking the electrical contact of the material by giving rise to undesirable side reactions between active material and electrolyte. This, in turn, would cause propagation and thickening of a so-called solid electrolyte interphase (SEI) layer and rapid deterioration of the capacity and the cycle life.

So far, a variety of design strategies has been developed for silicon which can be classified into three categories as follows: (i) downsizing of silicon (Fig. 1b) to feature sizes on the nanoscale (especially below the critical value) can provide materials surviving operation without mechanical fracture. Although discrete nanoparticles ${ }^{15,20,21}$, nanowires ${ }^{6,22,23}$, nanotubes ${ }^{24,25}$, nanosheets $^{26-28}$, and porous sponges ${ }^{29-31}$ have been employed, the issue of dynamic interfacing remains unsolved and, in many cases, even becomes more severe. Thereby, the contact between silicon and the adjacent conductive medium, specifically via single- or few-point physical binding, becomes ineffective and compromises cycle stability and rate capability; (ii) tailoring of the adjacent electrically conductive medium (Fig. 1c) by a combination of the downsized silicon with carbon nanostructures typically as yolk-shell or wire-in-tube structures. Thereby, the large volume change of silicon is accommodated and the interfacing with electrically conductive media and electrolyte is secured by the stable voids. Cycling stability is greatly improved, however, at the expense of rate capability. The reason for this shortcoming is that the single- or few-point physical binding retards and even obstructs charge transport from and to the silicon $^{32-44}$; (iii) covalent binding between the downsized silicon and adjacent electrically conductive media with the greatest potential to reduce accidental disconnection (Fig. 1d). Yet, this binding furnishes an inefficient point mode, incapable of substantially enhancing charge transport kinetics ${ }^{24,45-48}$. Moreover, it encounters risks of disruption upon electrolyte erosion. Taken all these conflicting requirements together, covalent binding of silicon appears as the method of choice for energy storage materials with large volume change, but is in need of conceptually new design principles.

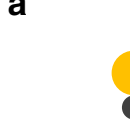

b
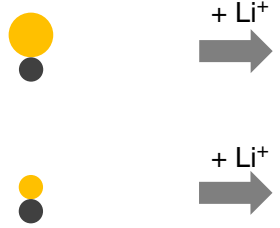

C
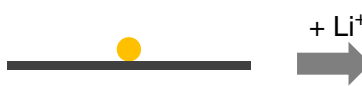

d
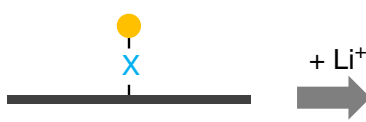

e
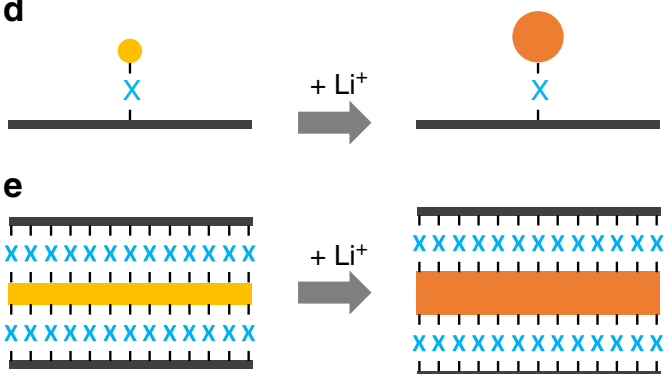
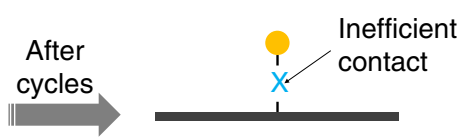

Ineffective

contact
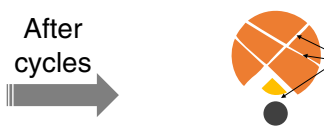

Ineffective

cycles

contact
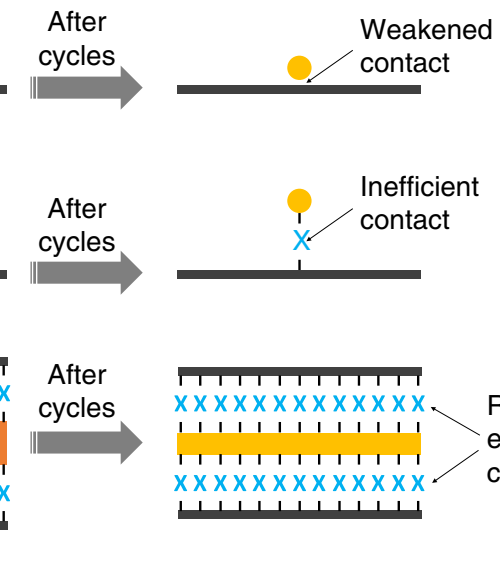

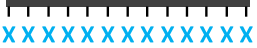

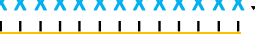

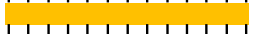
$x \times x \times x \times x \times x \times x=$

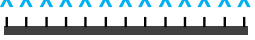

Robust \&

efficient

contact

Fig. 1 Binding strategies of silicon with electrically conductive media. a Point-mode physical binding of Si with a conventional conductive medium (e.g., carbon black). The large volume change causes pulverization of Si obstructing the contact and induces side reactions, as well as SEI propagation further deteriorating the contact. The ineffective binding or unbinding of Si results in poor cycling stability. b Downsizing of Si. Although resistant to mechanical fracture, the point-mode physical contact is similarly prone to become ineffective, hindering improvement in cycling and rate capability. c Tailoring of the adjacent conductive medium. With the Si/C combination to accommodate the volume change of Si, the cycling stability is improved; yet, this is at the expense of rate capability because the easily weakened point-mode physical binding is still adopted in most cases. $\mathbf{d}$ Manipulation of the binding between Si and the adjacent conductive medium. As the point mode of covalent binding is inefficient and unfavorable for the rate capability, the existing covalent binding still encounters risks of being disrupted due to the direct contact and propagating erosion of Si with the electrolyte. e Designing two-dimensional covalent binding for Si. This skin-like binding creates and more importantly, secures a robust and efficient contact between $\mathrm{Si}$ and $\mathrm{C}$ components, granting stable and fast electron/ion transport from/to the $\mathrm{Si}$ upon cycling. It is noteworthy that $\mathrm{Li}^{+}$is defined as lithium ions. 

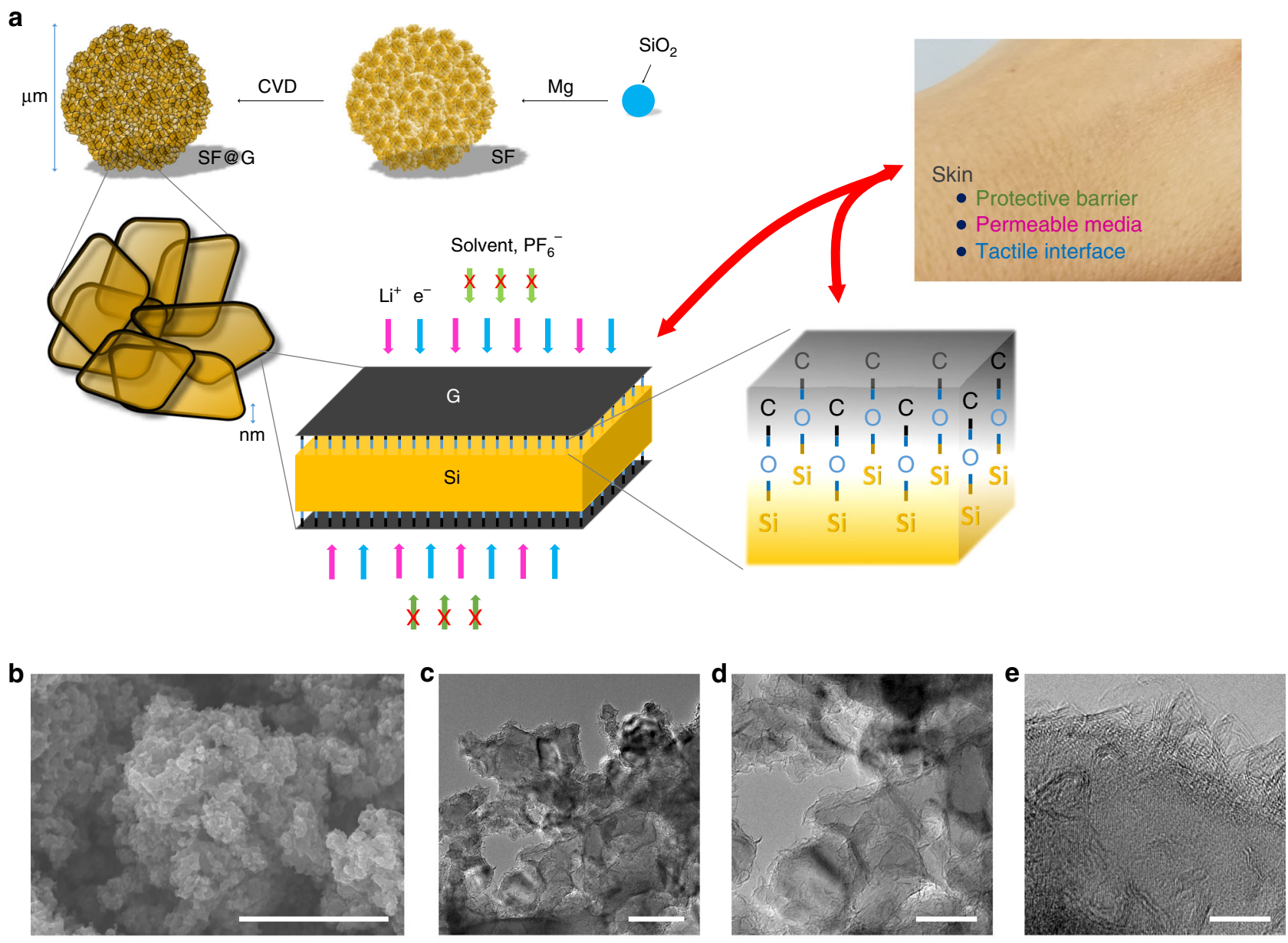

f
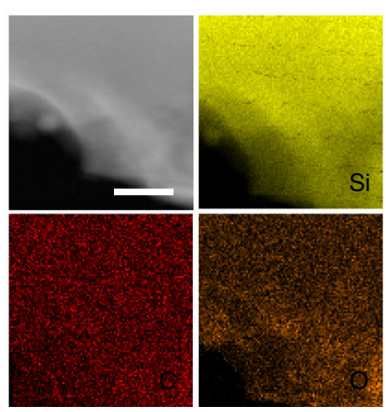

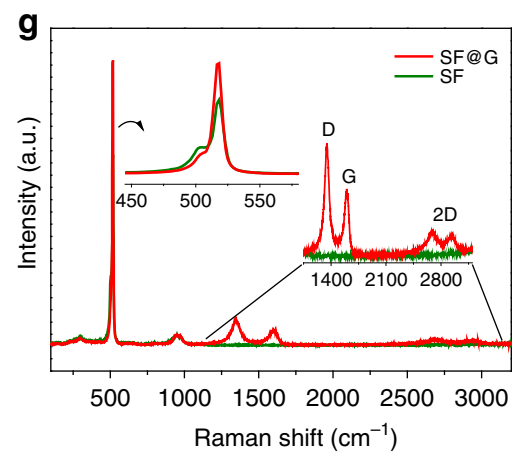

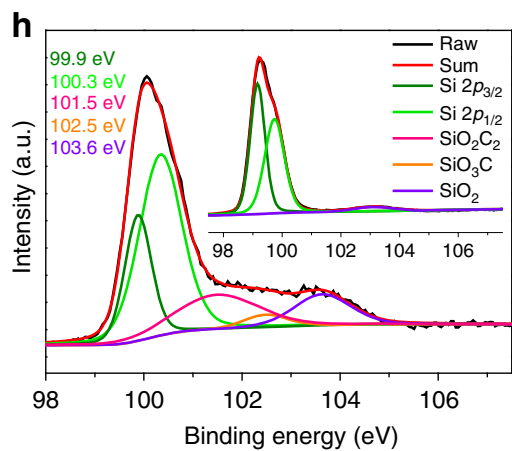

Fig. 2 Fabrication and characterization of SF@G. a Schematic of the fabrication process for SF@G. The synthesized SF@G features a two-dimensional covalently bound component interface, enabling stable and fast electron $\left(\mathrm{e}^{-}\right)$and lithium-ion $\left(\mathrm{Li}^{+}\right)$transport, while fundamentally blocking undesirable substances such as the electrolyte solvents $(\mathrm{EC}, \mathrm{DEC})$ and anions $\left(\mathrm{PF}_{6}{ }^{-}\right)$, mimicking the skin functions typically as a protective barrier, a permeable media, and a tactile interface. b SEM image of SF@G. c-e TEM images of SF@G. f Scanning transmission electron microscopy (STEM) and elemental mapping images of SF@G. $\mathbf{g}$ Raman spectra of SF@G and SF with magnified regions in the insets. h Si 2p XPS spectrum of SF@G along with that of SF in the inset, indicating the covalent binding at the Si/C component interface of SF@G. Scale bars, $2 \mu \mathrm{m}$ (b), $100 \mathrm{~nm}$ (c), $50 \mathrm{~nm}$ (d), $10 \mathrm{~nm}(\mathbf{e})$, and $100 \mathrm{~nm}$ (f).

Herein, we show a (skin-like) covalent encapsulation of silicon (Figs. 1e and 2a). As a proof-of-concept, two-dimensional covalently bound Si-C hybrid materials (namely, SF@G) are shown to exhibit stable, high-capacity, and high-rate lithium storage properties with respect to weight, volume, and area. Such a high level of integrated performance is markedly superior to previous literature studies. As a key issue, the binding between $\mathrm{Si}$ and $\mathrm{C}$ establishes a robust and efficient contact, and thus enables fast electron as well as ion transport from and to silicon. More importantly, as proven by interfacial morphology and chemical composition, this skin-like binding drastically changes the interface of silicon with the electrolyte and thus renders it stable upon cycling. Our material prototype unlocks the potential of the covalent encapsulation. Markedly different from previously reported concepts for silicon anodes where either physical binding or single- and few-point covalent binding have been adopted, our approach realizes a new binding mode between silicon and the adjacent electrically conductive media towards exploiting silicon-based lithium battery anodes with high integrated performance. 


\section{Results}

Fabrication and characterization. The SF@G material was synthesized by magnesium reduction of silicon dioxide to produce silicene flowers (SF) onto which graphene (G) was deposited by chemical vapor deposition (CVD) (Fig. 2a). Scanning electron microscopy (SEM), transmission electron microscopy (TEM), and elemental mapping images (Fig. 2b-f and Supplementary Fig. 1) reveal the morphology of SF@G. SF@G possesses a microsized hydrangea flower-like architecture composed of many interconnected nanoplates. On the surface of every nanoplate, graphene nanosheets (typically, 2 3 layers) are observed to be conformally deposited, in line with investigations of tap density, specific surface area, pore size distribution, as well as graphene morphology (Supplementary Figs. 2-4). The Raman spectrum (Fig. 2g) of SF@G displays an intense peak at $517 \mathrm{~cm}^{-1}$ with a shoulder at a lower frequency, confirming the intact nanoplates upon the CVD process ${ }^{27}$. Furthermore, the ratio of the D band to $\mathrm{G}$ band is estimated to be around 1.5, revealing the presence of pinholes, defects, and/or disordered domains in the deposited graphene favorable for ion transport (Fig. $2 \mathrm{~g}$ and Supplementary Fig. 5). In addition to the characteristic peaks of $\mathrm{Si}$ (99.9, $100.3 \mathrm{eV})$ and the native $\mathrm{SiO}_{2}$ layer $(103.6 \mathrm{eV})$, the X-ray photoelectron spectroscopy (XPS) spectrum of the $\mathrm{Si} 2 p$ region of SF@G exhibits two additional bands at 101.5 and $102.5 \mathrm{eV}$ (Fig. 2h), in line with $\mathrm{C} 1 \mathrm{~s}$ and $\mathrm{O} 1 \mathrm{~s}$ results (Supplementary Fig. 6). These peaks can be attributed to interfacial $\mathrm{Si}-\mathrm{O}-\mathrm{C}$ bonds between two-dimensional Si nanoplates and $\mathrm{G}$ nanosheets. Due to the conformal deposition of graphene and subsequent area-toarea contact between $\mathrm{Si}$ and $\mathrm{C}$ components, as verified by SEM and TEM observations, this covalent binding exists in a twodimensional mode. The covalent binding at the interface of twodimensional components can be described by a tentative chemical structure of SF@G (Supplementary Fig. 7). Also relevant is the absence of Si-O-C signals in the hydrofluoric acid (HF)-treated SF@G (SF@G-HF) (Supplementary Fig. 8). The "skin-formation" is suggested to be associated with the native silicon oxide layer on the Si nanoplates (Supplementary Fig. 9). Upon interfacing with the introduced hydrogen gas, the silicon oxide on Si nanoplates is partially reduced to $\mathrm{Si}-\mathrm{O}$ intermediates at high temperature. These intermediates are further combined with carbon species derived from methane, while additional supply of methane furnishes the deposition of graphene. Unless otherwise noted, the Si content in SF@G is about $88 \%$ as estimated by thermogravimetric analysis (Supplementary Fig. 10).

Electrochemical performance and kinetic characteristics. The achieved covalent, "layered" encapsulation of SF@G affords a remarkable battery performance (Figs. 3 and 4). As exhibited in Fig. 3a, b and Supplementary Fig. 11, SF@G demonstrates an improved initial Coulombic efficiency $(87 \%)$ and a rapid increase of stabilized Coulombic efficiency $>99 \%$, in sharp contrast with SF@G-HF and SF. This significantly enhanced Coulombic efficiency of SF@G depicts the interfacial difference between SF@G and SF@G-HF, although being made from the same components. Irreversible consumption of lithium and subsequent SEI formation can be rigorously prevented in SF@G. Although possessing the similar micro-sized architecture, SF@G also offers a dramatically improved cycling stability at a high rate of $2 \mathrm{~A} \mathrm{~g}^{-1}$ over 500 cycles compared with SF@G-HF and SF, delivering a high specific capacity that is more than five times higher than the theoretical capacity of graphite (Fig. 3c). The excellent cycling stability of SF@G is also verified by a prototype LFP//SF@G full cell device showing both stable cycling and high Coulombic efficiency (Fig. 3d). The capacities of SF@G and control electrodes at various rates (Fig. $4 \mathrm{a}, \mathrm{b}$ ) further demonstrate superb rate capability of the SF@G. The specific capacity of SF@G at rates of $0.8,2,4,8$, 12,16 , and $20 \mathrm{Ag}^{-1}$ is $2646,2194,1763,1389,1119,967$, and $812 \mathrm{mAh} \mathrm{g}^{-1}$, respectively. By comparison, both SF@G-HF and SF cannot deliver such a high capacity, especially at larger rates. Even after cycling at very high current rates of up to $20 \mathrm{~A} \mathrm{~g}^{-1}$, the capacity is still reproducable, firmly corroborating the high reversibility and cyclic stability of SF@G. As demonstrated in Fig. $4 c$, the rate performance of this two-dimensional covalently bound SF@G surpasses that of competing design concepts such as point-mode sulfur-bridged $\mathrm{Si} / \mathrm{C}^{45}$, point-mode oxygen-bridged $\mathrm{Si} / \mathrm{C}^{24}$, and noncovalently bound $\mathrm{Si} / \mathrm{C}^{37}$ (Fig. $4 \mathrm{c}$ ). Benefiting from the high gravimetric capacity and the high density of the material, the volumetric capacity of SF@G anodes is extraordinarily high (Fig. 4d, e). Considering the whole electrode volume as well as the volume change of 5.8 6.6\% upon cycling (Supplementary Fig. 12), SF@G exhibits a volumetric capacity of $2350 \mathrm{mAh} \mathrm{cm}^{-3}$ at a rate of $0.8 \mathrm{Ag}^{-1}$, which is more than four times that $\left(\sim 550 \mathrm{mAh} \mathrm{cm}^{-3}\right)$ of commercial graphite anodes. Even at high rates of $2,4,8,12,16$, and $20 \mathrm{~A} \mathrm{~g}^{-1}$, a volumetric capacity of 1952, 1547, 1202, 971, 869, and $694 \mathrm{mAh} \mathrm{cm}^{-3}$ is delivered, respectively, which is $54 \%, 74 \%, 65 \%, 699 \%, 1323 \%$, and $1442 \%$ of that of SF at the same rates. The achieved volumetric capacity of SF@G is markedly superior to previous results for different silicon anodes $19,21-24,29,31,32,34,39,40,43$ (Fig. 4e). Furthermore, the areal capacity of SF@G can be adjusted almost proportional to the mass loading of the active material, and a reversible areal capacity of $\sim 6 \mathrm{mAh} \mathrm{cm}^{-2}$ is reached at a mass loading of $2.48 \mathrm{mg} \mathrm{cm}^{-2}$ (Fig. 4f), much higher than that of a commercial LIB cell. In addition, the viability of SF@G is also characterized by a competitive energy density (Supplementary Table 1 and Supplementary Note 1). The high reversibility, high capacity, and high rate capability of SF@G reflect stable and fast electron and ion transport from and to the silicon, together with favorable lithium storage kinetics. These properties primarily stem from a robust and efficient contact between silicon and graphene at both sides of each nanoplate due to the covalent encapsulation and consequent two-dimensional tight binding between $\mathrm{Si}$ and $\mathrm{C}$, although the specific surface area may be an additional factor. This scenario is nicely supported by the Nyquist plots obtained from electrochemical impedance spectroscopy (EIS) (Supplementary Fig. 13) and further validated by the significantly improved $b$-values (defining the relation of peak current to sweep rate $)^{37}$ of both cathodic $(0.21 \mathrm{~V})$ and anodic $(0.35$ and $0.51 \mathrm{~V})$ peaks of SF@G in comparison with SF@G-HF and SF (Fig. 4g, Supplementary Fig. 14, and Supplementary Note 2).

Interfacial morphology and chemical composition upon cycling. To unravel the structural origin of the achieved performance, interfacial morphology and chemical composition of the cycled SF@G and control samples have been investigated. The SEM and TEM images of SF@G, SF@G-HF, and SF after 100 cycles are displayed in Fig. 5a-c and Supplementary Figs. 15 and 16. It is obvious that the SF@G retains the original flower-like architecture upon cycling, possessing a thin and smooth interfacial morphology. Different from that, the flower-like architecture of cycled SF@G-HF is blurred, with an abundance of rather rough SEI deposits filling up the gaps between SF@G-HF nanoplates. In case of cycled SF, the flower-like appearance is nearly completely deformed. The XPS results further depict interfacial SEI components of cycled SF@G, SF@G-HF, and SF (Fig. 5d-f and Supplementary Figs. 17 and 18). As shown in Fig. 5d, the C $1 s$ XPS spectrum of cycled SF@G reveals peaks assignable to $\mathrm{C}-\mathrm{C}, \mathrm{C}-\mathrm{O}$, and $\mathrm{O}=\mathrm{C}-\mathrm{O}(\mathrm{OCO})$ bonds, respectively. The $\mathrm{C}-\mathrm{O}$ and $\mathrm{OCO}$ bonds are confirmed by the detection of appropriate binding energy positions in the $\mathrm{O} 1 \mathrm{~s}$ spectrum of 

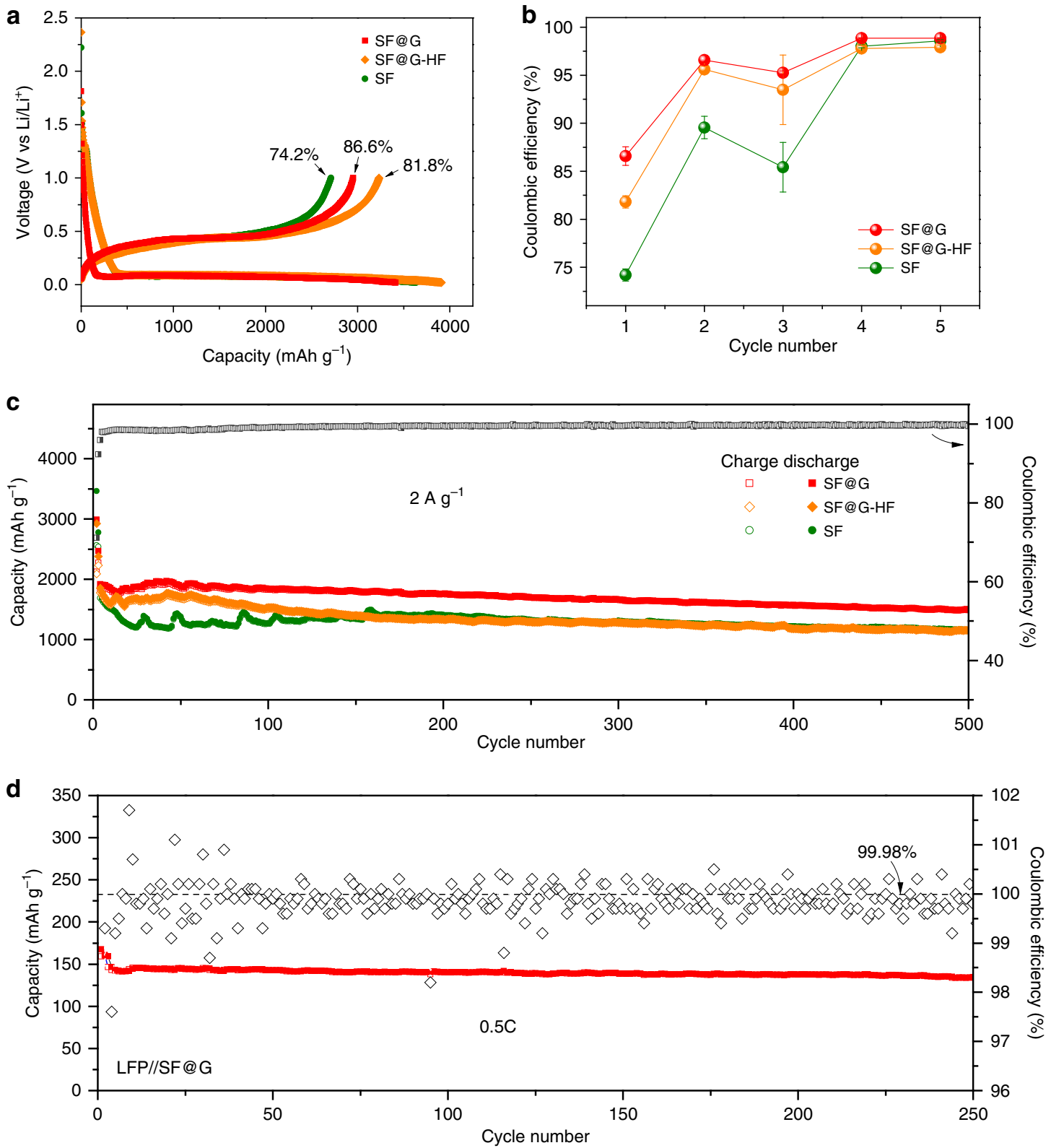

Fig. 3 Electrochemical characterization of SF@G upon cycling. a Charge-discharge profiles of SF@G, SF@G-HF, and SF at $0.2 \mathrm{Ag} \mathrm{g}^{-1}$ for the first cycle. b Coulombic efficiency of SF@G, SF@G-HF, and SF for initial five cycles. c Cycling performance of the SF@G and control electrodes over 500 cycles at $0.2 \mathrm{Ag}^{-1}$ for initial two cycles and $2 \mathrm{Ag}^{-1}$ for subsequent cycles. The Coulombic efficiency of SF@G is plotted on the secondary y-axis. $\mathbf{d}$ Reversible capacity and Coulombic efficiency vs. cycle plots of the LFP//SF@G full cell with SF@G as the anode and a commercial lithium iron phosphate (LFP) as the cathode (it is noteworthy that the capacity is based on the weight of active materials in the cathode).

SF@G, respectively. These results imply the presence of polyethylene oxide (PEO) and lithium alkoxides (ROLi) and carboxylates (ROCOLi) ${ }^{49-51}$ as typical SEI components in the cycled SF@G. Although the similar peaks corresponding to $\mathrm{C}-\mathrm{C}$ and $\mathrm{C}-\mathrm{O}$ bonds are still present, a prominent peak appears at $289.7 \mathrm{eV}$ in the cases of SF@G-HF and SF (Fig. 5e, f), attributable to carbonate-containing species (labeled as $\mathrm{CO}_{3}$ ). Their $\mathrm{O} 1 \mathrm{~s}$ spectra suggest the presence of $\mathrm{Li}_{2} \mathrm{CO}_{3}$ as the major SEI component in the cycled SF@G-HF and SF. These features clearly characterize the material interface, at which electrochemical reduction and consumption of the electrolyte solvents (ethylene carbonate (EC) and diethyl carbonate (DEC) in this work) occur

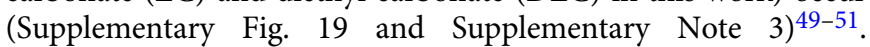
Although the SF@G-HF bears a similar electrolyte-interacting interface to that of SF reflecting the direct contact of Si with the electrolyte in both cases, SG@G is obviously different due to the presence of skin-like binding, in agreement with the Coulombic efficiency results. These findings are firmly supported by a series of additional peak assignments such as siloxane structures (R-Si-OR'), $\mathrm{LiF}$ and phosphorus compounds $\left(\mathrm{Li}_{x} \mathrm{PF}_{y}\right.$ and $\mathrm{Li}_{x} \mathrm{PO}_{y} \mathrm{~F}_{z}$ ) (see Supplementary Note 4 ).

The distinct difference in interfacial components is described further by the elemental compositions of the cycled samples (Fig. 5g). In the cycled SF@G, the majority is C ( 77 at\%), along with a low concentration of $\mathrm{O}$ and $\mathrm{Li}$, as well as an insignificant concentration of $\mathrm{F}$ and $\mathrm{P}$, proving the organic-dominated nature of SEI in SF@G. In comparison with SF@G, the cycled SF@G-HF and SF display similar atom concentrations corresponding to their inorganics-dominated SEI, where the concentration of $\mathrm{C}$ is substantially decreased to $\sim 37 \%$ and $\sim 32$ at $\%$, respectively, with 
a

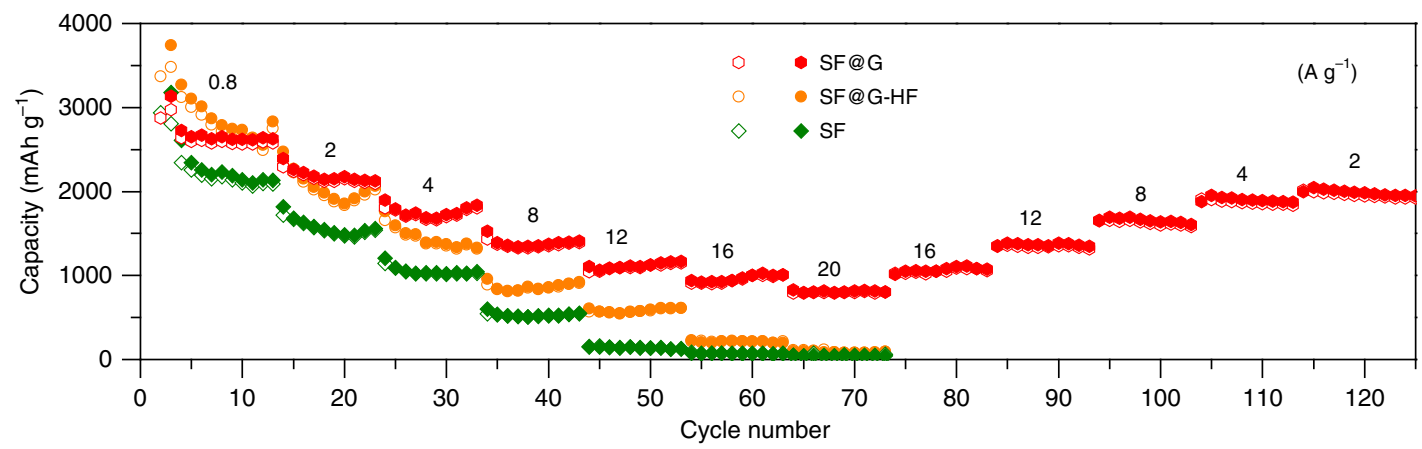

b

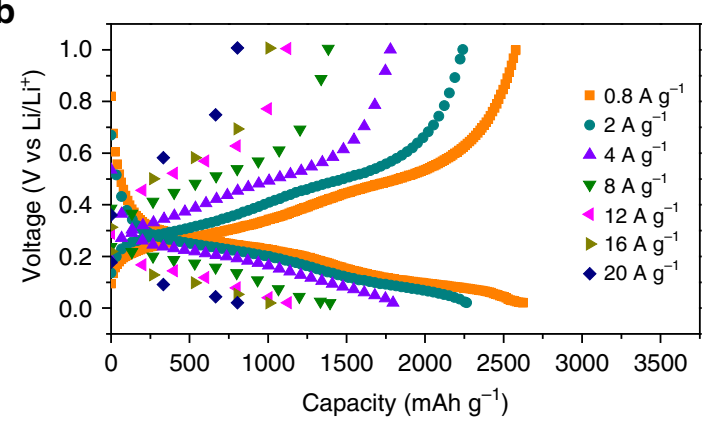

d

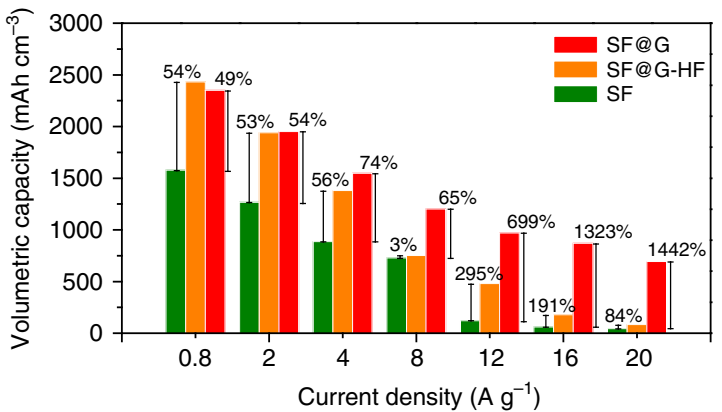

f

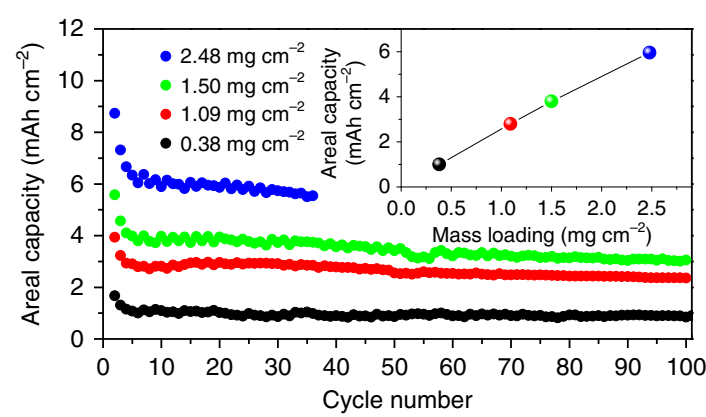

C 3500

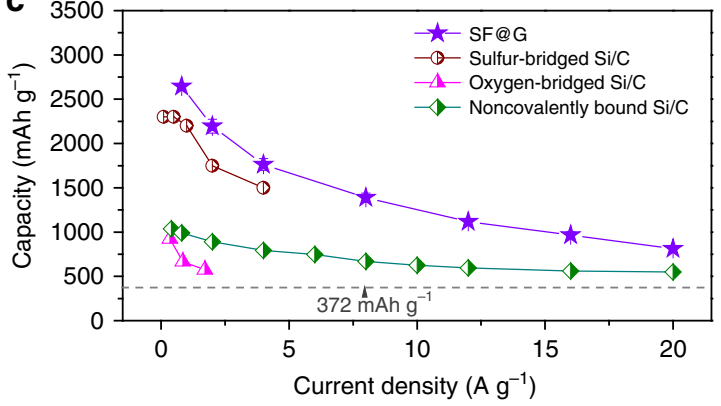

e

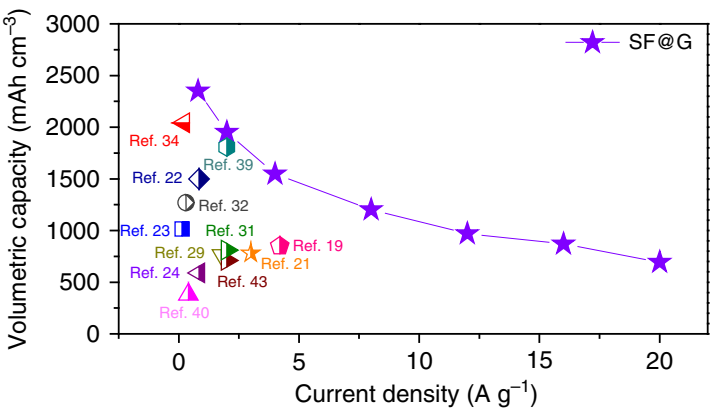

g

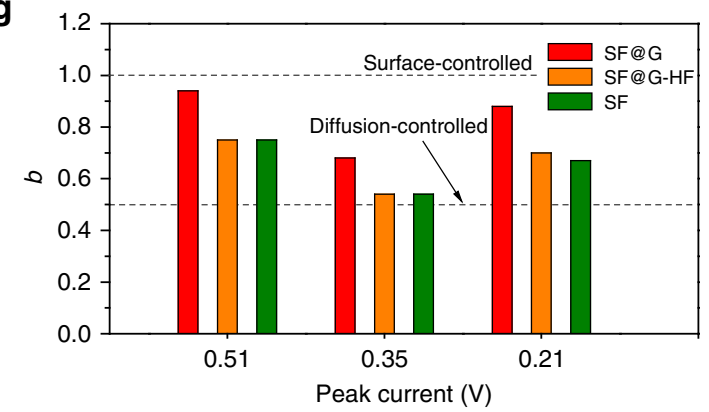

Fig. 4 Electrochemical characteristics of SF@G tested at different rates. a Capacity of SF@G, SF@G-HF, and SF cycled at different rates from 0.8 to 20 A g ${ }^{-1}$ (ten cycles for each rate).b Galvanostatic charge/discharge profiles of SF@G at various rates. c Capacity of SF@G at annotated rates, comparing with typical Si/C anodes with conventional interfacial binding modes as noted. d Volumetric capacity of SF@G, SF@G-HF, and SF at various rates. e Volumetric capacity at annotated current rates for SF@G with some representative Si anodes reported in the literatures as noted. $\mathbf{f}$ Areal capacity of SF@G at various active material mass loadings. $\mathbf{g}$ The $b$-value of anodic ( $0.51 \mathrm{~V}$ and $0.35 \mathrm{~V})$ and cathodic (0.21 V) peaks for SF@G, SF@G-HF, and SF, derived from cyclic voltammetry (CV) experiments at various sweep rates.

the concentration of $\mathrm{O}, \mathrm{Li}, \mathrm{F}, \mathrm{P}$, and $\mathrm{Si}$ significantly increased. The distinctly high concentration of $\mathrm{O}$ and $\mathrm{Li}$ in both SF@G-HF and SF points toward $\mathrm{Li}_{2} \mathrm{CO}_{3}$ as a major component. As schematically shown in Fig. 5h, the main interfacial ingredients of cycled SF@G-HF and SF consistently include $\mathrm{Li}_{2} \mathrm{CO}_{3}, \mathrm{LiF}, \mathrm{Li}_{x} \mathrm{PF}_{y}$, $\mathrm{Li}_{x} \mathrm{PO}_{y} \mathrm{~F}_{z}$, and R-Si-OR'; the interface of cycled SF@G mostly consists of organic species (e.g., PEO, ROLi, and ROCOLi), with an almost negligible amount of inorganic materials. After washing with 5\% hydrochloric acid $(\mathrm{HCl})$, the cycled SF@G exhibits an analogous interfacial morphology and elemental composition (e.g., C, O) to its unwashed one, whereas the cycled SF@G-HF and SF show a scenario totally different from unwashed counterparts (Supplementary Figs. 17 and 20-22), reflecting stable characteristics of the SF@G interface. The differences 


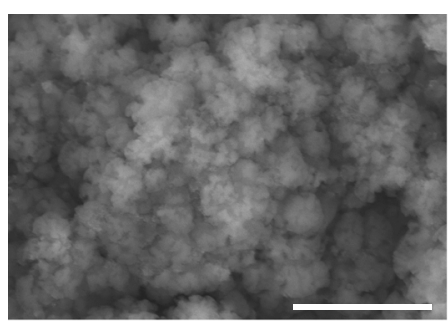

b

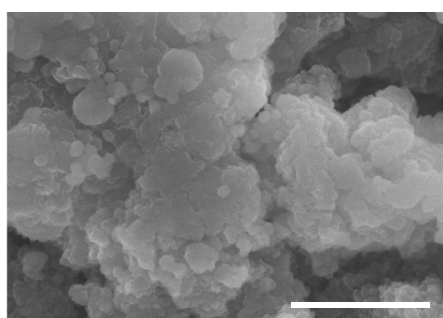

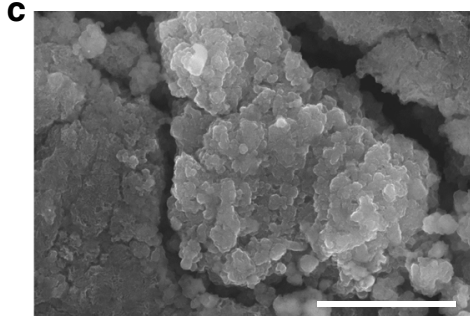

d

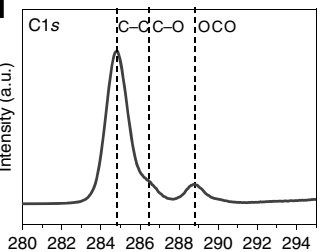

Binding energy (eV)

e

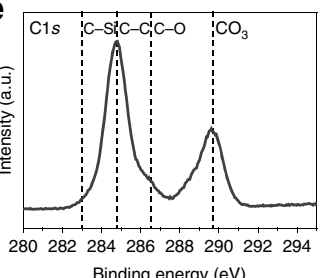

f

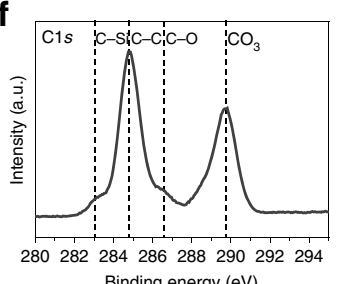

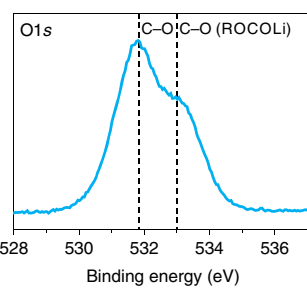
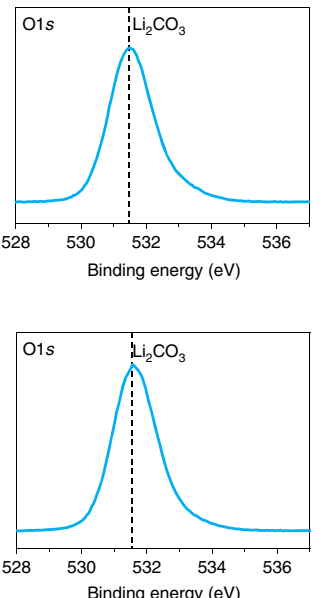
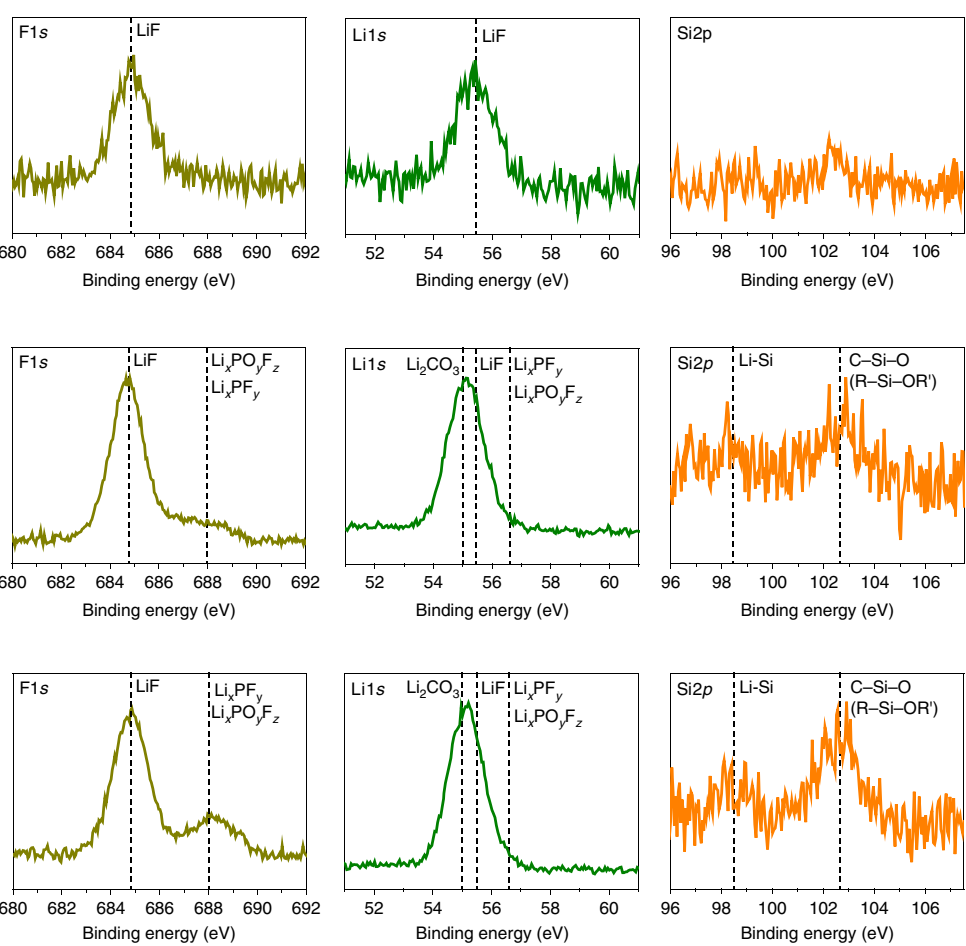

g

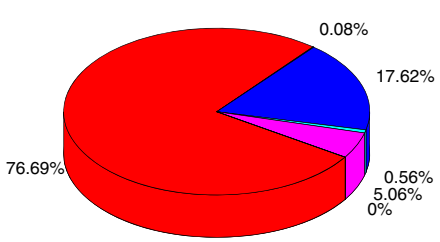

SF@G

h

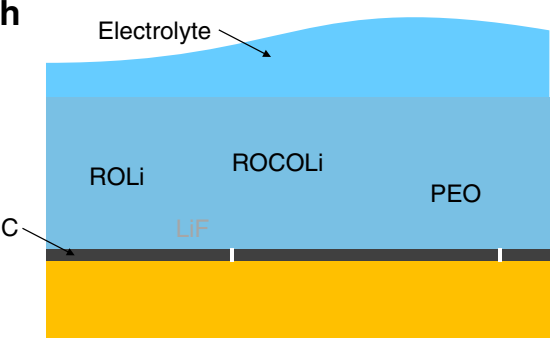

SF@G
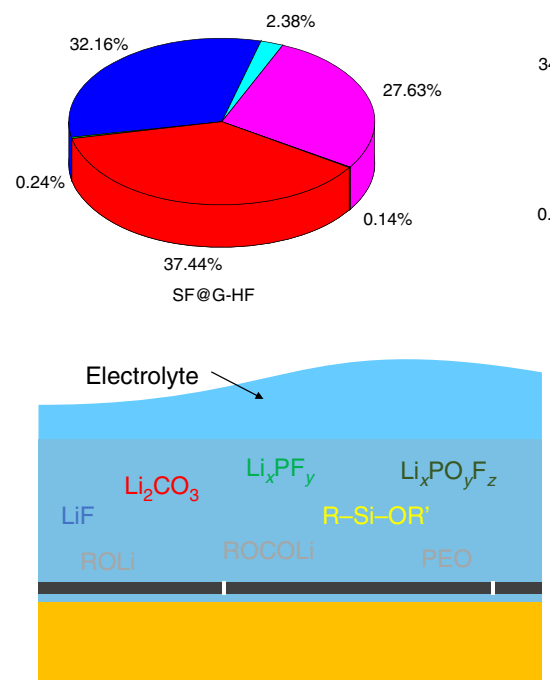

SF@G-HF
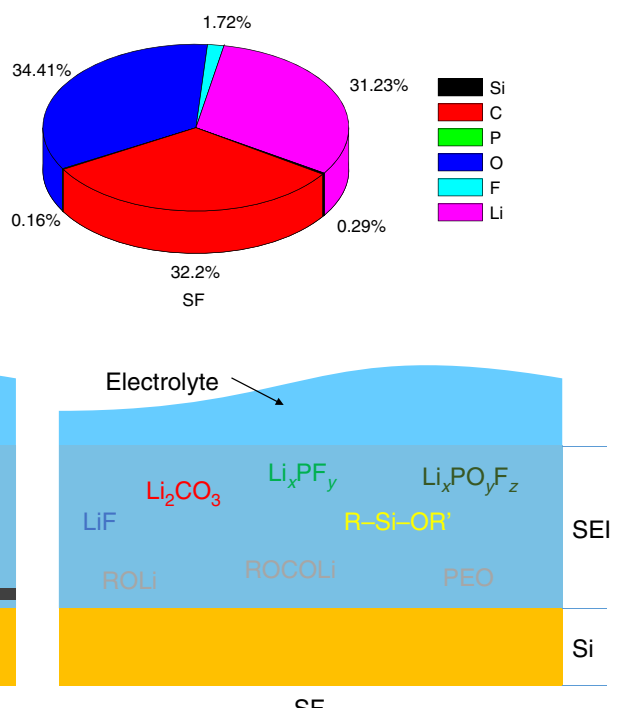

SF

Fig. 5 Interfacial morphology and chemical composition after cycling. a-c SEM images of (a) SF@G, (b) SF@G-HF, and (c) SF after 100 cycles. d-f C 1S, O 1s, F 1s, Li 1s, and Si 2p XPS spectra of (d) cycled SF@G, (e) cycled SF@G-HF, and (f) cycled SF. g Interfacial atom concentrations of cycled SF@G, SF@GHF, and SF. h Schematic description of the interface of cycled SF@G, SF@G-HF, and SF, showing distinctly different components as the majority of cycled SF@G and control samples. It is noteworthy that the components annotated in gray represent the minority and the SEI thickness is not scaled. Scale bars, $2 \mu \mathrm{m}(\mathbf{a}-\mathbf{c})$. 
discussed above are also revealed by elemental compositions obtained from EDX analyses (Supplementary Fig. 23 and Supplementary Note 5). This interfacial contact stability is validated by EIS results (Supplementary Fig. 24). It is noteworthy that the covalent binding present in SF@G is well retained, whereas its two-dimensional hybrid structure persists upon cycling (Supplementary Figs. 20, 25, and 26, and Supplementary Note 6). It should be noted as well that this structural and interfacial stabilization, combined with minimized electrode thickness variations (Supplementary Fig. 12), point toward better accommodation of the volume change of silicon in SF@G. This can be attributed to the two-dimensional character (mimicking the behavior of a planar thin film) and three-dimensional spatial orientation (creating free spaces to accommodate the volume change) of the involved nanoplates ${ }^{27}$. The similar interfacial morphology and chemical composition of cycled SF@G-HF and SF disclose an insufficient ability of the carbon hybridized with silicon in a two-dimensional noncovalent manner for blocking the direct contact of $\mathrm{Si}$ with the electrolyte. Specifically, in the case of SF@G-HF, graphene (G) cannot effectively inhibit undesirable side reactions between $\mathrm{Si}$ and electrolyte and consequent SEI propagation upon cycling, even if a sandwich-like hybrid structure is adopted to mitigate the structural and interfacial variation of $\mathrm{Si}$ upon lithiation and delithiation. One expects that the electrolyte penetrates through pinholes and/or defects of $G$ and interacts with the dynamic Si interface. By contrast, the marked difference in interfacial morphology and composition of cycled SF@G provides firm evidence of the critically important role of skin-like covalent binding in our concept to fundamentally block direct contact of Si with the electrolyte, confer a thin and stable SEI, and maintain the as-established contact during cycling.

\section{Discussion}

In conclusion, a design strategy for skin-like covalent encapsulation of silicon electrodes is developed to address the issue of its large volume change. The proof-of-concept of two-dimensional, covalently bound silicon-carbon hybrids exhibits stable highcapacity and high-rate lithium storage performances when referred to weight, volume and area. These outstanding results are superior to previous investigations. Markedly distinct from existing techniques of battery fabrication, the involved twodimensional, covalent binding creates a robust and efficient contact between the silicon and electrically conductive media, enabling stable and fast electron as well as ion transport from and to the silicon. As certified by distinctive interfacial morphology and binding modes between elements, this encapsulation rigorously blocks the direct contact of silicon with the electrolyte and changes the material interface, making the contacts persistent to cycling. Combined with a cost-effective raw material and a simple, facile, and scalable manufacturing process, the study opens a new and viable avenue to stabilize silicon without sacrificing parameters including capacity and rate capability. Further, our work can stimulate protocols for the rational design and mass production of other advanced energy materials to be used in lithium storage and beyond.

\section{Methods}

Preparation of SF@G. The SF@G was fabricated by magnesium reduction and CVD processes as schematically shown in Fig. 2a. Briefly, the freshly prepared SF, through magnesium reduction of silicon dioxide $\left(\mathrm{SiO}_{2}\right)$ as previously reported ${ }^{27}$, was placed in a quartz vessel, which was heated at a ramp rate of $5^{\circ} \mathrm{C} \mathrm{min}^{-1}$ to $1050{ }^{\circ} \mathrm{C}$ in a horizontal tube furnace under argon/hydrogen $\left(\mathrm{Ar} / \mathrm{H}_{2} ; 1: 1\right)$ atmosphere. The Ar flow was then turned off, the $\mathrm{H}_{2}$ flow was maintained at 100 standard cubic centimeters (s.c.c.m.), and 100 s.c.c.m. of methane $\left(\mathrm{CH}_{4}\right)$ was introduced into the reaction tube and kept for $10 \mathrm{~min}$. Cooling of the sample to room temperature under the protection of $\mathrm{Ar}$ and $\mathrm{H}_{2}$ furnished SF@G. The SF@GHF control sample was obtained by immersing the as-prepared SF@G in 5\% HF solution for a defined time (typically, $1 \mathrm{~h}$ ) to break the covalent binding at the $\mathrm{Si} / \mathrm{C}$ interface, followed by repeated washing and subsequent drying. In addition, graphene was obtained by removing the silicon of SF@G with 5\% sodium hydroxide $(\mathrm{NaOH})$ aqueous solution under $80^{\circ} \mathrm{C}$

Material characterization. The morphology and structure of all samples were investigated by FE-SEM (Hitachi S4800) and FE-TEM (FEI Tecnai G2 20 STWIN and Tecnai G2 F20 U-TWIN). Raman spectra were collected using a Renishaw inVia Raman microscope with a laser wavelength of $514.5 \mathrm{~nm}$. XPS measurements were performed on an ESCALAB250Xi apparatus with an Al Ka X-ray source. Before the XPS measurements, the cycled samples were washed repeatedly with fresh dimethyl carbonate to remove the residual electrolyte; in some cases, the cleaned samples were subjected to further washing with $5 \% \mathrm{HCl}$ to remove most unstable SEI components of the samples if there exist. Nitrogen adsorption/desorption isotherms were measured at $77 \mathrm{~K}$ with an ASAP 2020 physisorption analyzer The Brunauer-Emmett-Teller method and Barrett-Joyner-Halenda model were utilized to estimate the specific surface area and pore size distribution, respectively.

Electrochemical characterization. The working electrodes were made by a typical slurry method with active materials (SF@G, SF@G, or SF), conductive additive (Super P, Alfa Aesar), and polyacrylic acid (weight-average molecular weight of 240,000, Alfa Aesar) binder at a mass ratio of 8:1:1. Unless otherwise specified, the typical mass loading of active materials was $1.0 \sim 2.5 \mathrm{mg} \mathrm{cm}^{-1}$. All the electrodes were degassed in vacuum at $60^{\circ} \mathrm{C}$ for at least $2 \mathrm{~h}$ before use. Two-electrode CR2032 coin-type half-cells were assembled in an argon-filled glove box $(<0.1$ p.p.m. of oxygen and water) with lithium foil as the counter electrode. The electrolyte was 1.0 $\mathrm{M} \mathrm{LiPF}_{6}$ in $1: 1(\mathrm{v} / \mathrm{v}) \mathrm{EC} / \mathrm{DEC}$ with $5 \%$ fluoroethylene carbonate and the separator was porous polypropylene films (Celgard 2400). The full cell was designed with a N/P ratio of ca. 1.05. The cathode electrodes were fabricated by mixing commercial lithium iron phosphate (LFP), carbon black, and polyvinylidene fluoride in $N$-methyl-2-pyrrolidone at a mass ratio of 8:1:1, casting, and drying in vacuum at $80^{\circ} \mathrm{C}$ for at least $2 \mathrm{~h}$. Coin-type full cells with the fabricated LFP cathodes and SF@G anodes were assembled in a glove box filled with argon gas. The electrolytes and separators in the full cells were the same as those used in the half cells. Electrochemical tests were carried out in the voltage window between 2.5 and $4.2 \mathrm{~V}$. The cycling and rate capability tests were performed using a CT2001A battery program controlling test system within the voltage range of $0.02-1.0 \mathrm{~V}$. Cyclic voltammetry was carried out in the potential range of $0.02-1.0 \mathrm{~V}$ at various rates $\left(0.1 \sim 1.0 \mathrm{mV} \mathrm{s}^{-1}\right)$ with a CHI660D electrochemical station. Unless otherwise specified, all electrochemical measurements were undertaken at room temperature in half-cells, the capacity reported was based on the total weight of active materials in the working electrode, as well as the annotated cycling conditions. The volumetric capacity was calculated as product of gravimetric capacity and packing density of the electrode, and the coulombic efficiency calculated using the ratio of delithiation capacity to lithiation capacity.

\section{Data availability}

The data supporting the findings of this study are available from the corresponding author upon reasonable request.

Received: 15 June 2019; Accepted: 14 July 2020; Published online: 31 July 2020

\section{References}

1. Schmuch, R., Wagner, R., Hörpel, G., Placke, T. \& Winter, M. Performance and cost of materials for lithium-based rechargeable automotive batteries. Nat. Energy 3, 267-278 (2018).

2. Kang, B. \& Ceder, G. Battery materials for ultrafast charging and discharging Nature 458, 190-193 (2009).

3. Armand, M. \& Tarascon, J.-M. Building better batteries. Nature 451, 652-657 (2008).

4. Arico, A. S., Bruce, P., Scrosati, B., Tarascon, J.-M. \& van Schalkwijk, W. Nanostructured materials for advanced energy conversion and storage devices. Nat. Mater. 4, 366--3377 (2005).

5. Tarascon, J.-M. \& Armand, M. Issues and challenges facing rechargeable lithium batteries. Nature 414, 359-367 (2001).

6. Chan, C. K. et al. High-performance lithium battery anodes using silicon nanowires. Nat. Nanotechnol. 3, 31-35 (2008).

7. Lin, D., Liu, Y. \& Cui, Y. Reviving the lithium metal anode for high-energy batteries. Nat. Nanotechnol. 12, 194-206 (2017).

8. Li, B., Li, S., Xu, J. \& Yan, S. A new configured lithiated silicon-sulfur battery built on 3D graphene with superior electrochemical performances. Energy Environ. Sci. 9, 2025-2030 (2016).

9. Li, X. \& Zhi, L. Graphene hybridization for energy storage applications. Chem. Soc. Rev. 47, 3189-3216 (2018). 
10. Raccichini, R., Varzi, A., Passerini, S. \& Scrosati, B. The role of graphene for electrochemical energy storage. Nat. Mater. 14, 271-279 (2015).

11. Zhang, C., Lv, W., Tao, Y. \& Yang, Q.-H. Towards superior volumetric performance: design and preparation of novel carbon materials for energy storage. Energy Environ. Sci. 8, 1390-1403 (2015).

12. Wu, H. \& Cui, Y. Designing nanostructured Si anodes for high energy lithium ion batteries. Nano. Today 7, 414-429 (2012).

13. Moshtev, R. \& Johnson, B. State of the art of commercial Li ion batteries. J. Power Sources 91, 86-91 (2000).

14. Graetz, J., Ahn, C. C., Yazami, R. \& Fultz, B. Highly reversible lithium storage in nanostructured silicon. Electrochem. Solid State Lett. 6, A194-A197 (2003).

15. Kasavajjula, U., Wang, C. \& Appleby, A. J. Nano- and bulk-silicon-based insertion anodes for lithium-ion secondary cells. J. Power Sources 163, 1003-1039 (2007).

16. Piper, D. M. et al. Stable silicon-ionic liquid interface for next-generation lithium-ion batteries. Nat. Commun. 6, 6230 (2015).

17. Yu, G. et al. Stable Li-ion battery anodes by in-situ polymerization of conducting hydrogel to conformally coat silicon nanoparticles. Nat. Commun. 4, 1943 (2013).

18. $\mathrm{Wu}, \mathrm{H}$. et al. Stable cycling of double-walled silicon nanotube battery anodes through solid-electrolyte interphase control. Nat. Nanotechnol. 7, 310-315 (2012).

19. Kovalenko, I. et al. A major constituent of brown algae for use in highcapacity Li-ion batteries. Science 334, 75-79 (2011).

20. Liu, X. H. et al. Size-dependent fracture of silicon nanoparticles during lithiation. ACS Nano 6, 1522-1531 (2012).

21. Magasinski, A. et al. High-performance lithium-ion anodes using a hierarchical bottom-up approach. Nat. Mater. 9, 353-358 (2010).

22. Wang, B. et al. High volumetric capacity silicon-based lithium battery anodes by nanoscale system engineering. Nano Lett. 13, 5578-5584 (2013).

23. Jeong, $\mathrm{S}$. et al. Etched graphite with internally grown $\mathrm{Si}$ nanowires from pores as an anode for high density Li-ion batteries. Nano Lett. 13, 3403-3407 (2013).

24. Sun, C.-F. et al. Interfacial oxygen stabilizes composite silicon anodes. Nano Lett. 15, 703-708 (2015).

25. Park, M.-H. et al. Silicon nanotube battery anodes. Nano Lett. 9, 3844-3847 (2009).

26. Liu, J., Yang, Y., Lyu, P., Nachtigall, P. \& Xu, Y. Few-layer silicene nanosheets with superior lithium-storage properties. Adv. Mater. 30, 1800838 (2018).

27. Zhang, X. et al. Silicene flowers: A dual stabilized silicon building block for high-performance lithium battery anodes. ACS Nano 11, 7476-7484 (2017).

28. Ryu, J., Hong, D., Choi, S. \& Park, S. Synthesis of ultrathin Si nanosheets from natural clays for lithium-ion battery anodes. ACS Nano 10, 2843-2851 (2016).

29. Xiao, Q. et al. Inward lithium-ion breathing of hierarchically porous silicon anodes. Nat. Commun. 6, 8844 (2015).

30. Li, X. et al. Mesoporous silicon sponge as an anti-pulverization structure for high-performance lithium-ion battery anodes. Nat. Commun. 5, 4105 (2014).

31. Ge, M. et al. Large-scale fabrication, 3D tomography, and lithium-ion battery application of porous silicon. Nano Lett. 14, 261-268 (2014).

32. Liu, N. et al. A pomegranate-inspired nanoscale design for large-volumechange lithium battery anodes. Nat. Nanotechnol. 9, 187-192 (2014).

33. Wang, B. et al. Contact-engineered and void-involved silicon/carbon nanohybrids as lithium-ion-battery anodes. Adv. Mater. 25, 3560-3565 (2013).

34. Wang, J. et al. Shell-protective secondary silicon nanostructures as pressureresistant high-volumetric-capacity anodes for lithium-ion batteries. Nano Lett. 18, 7060-7065 (2018).

35. Shang, H. et al. Low-temperature growth of all-carbon graphdiyne on a silicon anode for high-performance lithium-ion batteries. Adv. Mater. 30, 1801459 (2018).

36. Chen, S., Shen, L., van Aken, P. A., Maier, J. \& Yu, Y. Dual-functionalized double carbon shells coated silicon nanoparticles for high performance lithium-ion batteries. Adv. Mater. 29, 1605650 (2017).

37. Wang, B. et al. Approaching the downsizing limit of silicon for surfacecontrolled lithium storage. Adv. Mater. 27, 1526-1532 (2015).

38. Son, I. H. et al. Silicon carbide-free graphene growth on silicon for lithium-ion battery with high volumetric energy density. Nat. Commun. 6, 7393 (2015).

39. Zhou, M. et al. High-performance silicon battery anodes enabled by engineering graphene assemblies. Nano Lett. 15, 6222-6228 (2015).

40. Ji, J. et al. Graphene-encapsulated Si on ultrathin-graphite foam as anode for high capacity lithium-ion batteries. Adv. Mater. 25, 4673-4677 (2013).

41. Zhou, X., Yin, Y.-X., Wan, L.-J. \& Guo, Y.-G. Self-assembled nanocomposite of silicon nanoparticles encapsulated in graphene through electrostatic attraction for lithium-ion batteries. Adv. Energy Mater. 2, 1086-1090 (2012).

42. Ryu, J. et al. Mechanical mismatch-driven rippling in carbon-coated silicon sheets for stress-resilient battery anodes. Nat. Commun. 9, 2924 (2018).
43. Zhang, R. et al. Highly reversible and large lithium storage in mesoporous $\mathrm{Si} / \mathrm{C}$ nanocomposite anodes with silicon nanoparticles embedded in a carbon framework. Adv. Mater. 26, 6749-6755 (2014).

44. Ogata, K. et al. Evolving affinity between Coulombic reversibility and hysteretic phase transformations in nanostructured silicon-based lithium-ion batteries. Nat. Commun. 9, 479 (2018).

45. Hassan, F. M. et al. Evidence of covalent synergy in silicon-sulfur-graphene yielding highly efficient and long-life lithium-ion batteries. Nat. Commun. $\mathbf{6}$, 8597 (2015)

46. Martin, C. et al. Chemical coupling of carbon nanotubes and silicon nanoparticles for improved negative electrode performance in lithium-ion batteries. Adv. Funct. Mater. 21, 3524-3530 (2011).

47. Martin, C. et al. Graphite-grafted silicon nanocomposite as a negative electrode for lithium-ion batteries. Adv. Mater. 21, 4735-4741 (2009).

48. Osaka, T., Nara, H., Momma, T. \& Yokoshima, T. New Si-O-C composite film anode materials for LIB by electrodeposition. J. Mater. Chem. A 2, 883 (2014).

49. Schroder, K. W., Celio, H., Webb, L. J. \& Stevenson, K. J. Examining solid electrolyte interphase formation on crystalline silicon electrodes: influence of electrochemical preparation and ambient exposure conditions. J. Phys. Chem. C. 116, 19737-19747 (2012).

50. Yen, Y. C., Chao, S.-C., Wu, H.-C. \& Wu, N.-L. Study on solid-electrolyteinterphase of $\mathrm{Si}$ and C-coated $\mathrm{Si}$ electrodes in lithium cells. J. Electrochem. Soc. 156, A95-A102 (2009).

51. Chan, C. K., Ruffo, R., Hong, S. S. \& Cui, Y. Surface chemistry and morphology of the solid electrolyte interphase on silicon nanowire lithium-ion battery anodes. J. Power Sources 189, 1132-1140 (2009).

\section{Acknowledgements}

We acknowledge support from the National Natural Science Foundation of China (Grant Number 51425302) and Youth Innovation Promotion Association CAS (Number 2016033)

\section{Author contributions}

X.L. and L.Z. proposed and supervised the project. X.Z. designed and carried out the experiments. D.W., X.Q., Y.M., and D.K. contributed to the sample characterization and provided some experimental assistance. X.Z., X.L., and L.Z. discussed and analyzed the results. K.M. provided some suggestions and co-wrote the paper.

\section{Competing interests}

The authors declare no competing interests.

\section{Additional information}

Supplementary information is available for this paper at https://doi.org/10.1038/s41467020-17686-4.

Correspondence and requests for materials should be addressed to X.L. or L.Z.

Peer review information Nature Communications thanks Ken Ogata, and the other, anonymous, reviewer(s) for their contribution to the peer review of this work. Peer reviewer reports are available.

Reprints and permission information is available at http://www.nature.com/reprints

Publisher's note Springer Nature remains neutral with regard to jurisdictional claims in published maps and institutional affiliations.

\footnotetext{
Open Access This article is licensed under a Creative Commons Attribution 4.0 International License, which permits use, sharing adaptation, distribution and reproduction in any medium or format, as long as you give appropriate credit to the original author(s) and the source, provide a link to the Creative Commons license, and indicate if changes were made. The images or other third party material in this article are included in the article's Creative Commons license, unless indicated otherwise in a credit line to the material. If material is not included in the article's Creative Commons license and your intended use is not permitted by statutory regulation or exceeds the permitted use, you will need to obtain permission directly from the copyright holder. To view a copy of this license, visit http://creativecommons.org/ licenses/by/4.0/.
}

(C) The Author(s) 2020 